\title{
Benchmarking atomic data for astrophysics: Fe XXIV
}

\author{
G. Del Zanna
}

University College London, MSSL, Holmbury St. Mary Dorking Surrey RH5 6NT, UK

e-mail: gdz@mssl.ucl.ac.uk

Received 9 December 2004 / Accepted 11 October 2005

\section{ABSTRACT}

Fe XXIV produces strong L-shell $(n=3,4,5 \rightarrow 2)$ spectral lines in the X-rays. These lines have been observed in solar flares, in laboratory spectra, and in a variety of astrophysical sources with e.g. the Chandra and XMM-Newton satellites. In this paper atomic data for Fe XXIV L-shell emission are benchmarked against experimental data. The work focuses on reviewing and assessing previous line identifications on a quantitative basis, paying particular attention to the most important lines for laboratory and astrophysical applications. Previous identifications are confirmed, but many lines turn out to be significantly blended. The agreement between theoretical and observed data in terms of wavelengths, line intensities and level lifetimes is good, within the experimental uncertainties. Fe XXIV L-shell lines can be used to measure electron densities in laboratory plasmas and temperatures for a wide range of astrophysical sources.

Key words. atomic data - line: identification - Sun: flares - techniques: spectroscopic

\section{Introduction}

This paper is one in a series which aims to benchmark the latest atomic data against high-resolution spectroscopic data, and in particular to discuss line identifications and blends, and suggest the best spectral lines to be used for plasma diagnostics. The approach is practical since it focuses only on the lines that are predicted to be brightest in laboratory spectra and in a wide range of astrophysical conditions. For a description of the methods and goals see Paper I (Del Zanna et al. 2004).

In this paper Fe XXIV $n=3,4,5 \rightarrow 2$ (L-shell) emission is considered. Fe XXIV L-shell emission is prominent in solar flare spectra (see, e.g., Neupert et al. 1967), and in laboratory plasmas (see, e.g., Boiko et al. 1978). In recent years, highresolution XUV spectroscopic observations by the Chandra and XMM-Newton satellites have also shown that a wealth of "hot" astrophysical sources produce strong Fe XXIV L-shell emission.

Li-like iron is such a simple ion that all atomic structure calculations performed in the past produce energies and oscillator strengths that are very accurate, when compared to other ions. It is no surprise then that most of the Fe XXIV energy levels have been previously identified. It should be noted, however, that previous identifications of Fe XXIV were simply based on approximate comparisons between oscillator strengths and observed spectra, and not on a quantitative basis. The first aim of this paper is to confirm, whenever possible, these identifications and to highlight the presence of blends. The second aim is to show that Fe XXIV L-shell emission can provide density (for high-density laboratory plasmas) and temperature (for astrophysical sources) diagnostics. These diagnostics were previously known but have not been applied to spectral observations in the literature.

Fe XXIV L-shell spectral lines fall in the 6-12 $\AA$ range, which is, even in laboratory spectra with the highest spectral resolution, densely packed with hundreds of transitions from different ionization stages of iron and other elements. A large number of lines are still either unidentified or have a questionable identification. This paper is part of an on-going benchmark work on each ion separately, by checking intensities and wavelengths of the brightest lines, and at the same time consider all ions that can possibly blend the observed lines. The identifications of Fe XXIII L-shell lines and their diagnostic use has already been studied in detail by Del Zanna et al. (2005), using the recent scattering calculations of Chidichimo et al. (2005).

Section 2 describes the experimental data that have been used in the benchmark. Section 3 describes the procedures and the atomic data adopted. Section 4 presents the results while Sect. 5 draws the conclusions.

\section{Observations of FeXXIV lines}

The first observations of Fe XXIV lines in solar flares were made with the OSO-III satellite which observed the 1.3-20 A region and were reported by Neupert et al. (1967). They contained $n=3,4 \rightarrow 2$ L-shell spectral lines. Doschek et al. (1972) identified a few Fe XXIV $n=4 \rightarrow 2$ transitions at $\lambda \simeq 8 \AA$ from OSO-6 solar spectra, while Doschek et al. (1973) provided a more extended line list including $n=3 \rightarrow 2$ lines, also recorded by OSO-6. Neupert et al. (1973) presented OSO-5 spectra of solar flares in the 6-25 A region. 
Various laboratory iron spark spectra were produced in the attempt to identify the iron L-shell emission, but it was only when laser spectra were produced that the first identifications based on laboratory data were possible. Many identifications of $n=3,4,5 \rightarrow 2$ lines were suggested using laser spectra of excellent quality and published in a series of papers (see the review in Boiko et al. 1978, and references therein). These identifications were further confirmed by Fawcett et al. (1979) also using laser spectra.

The Boiko et al. (1978) line lists are still the best to study highly excited iron ions for a number of reasons. The authors provided line intensities, which were corrected for the film response and the filter absorption, although not for the reflectivity of the mica crystal. Hence, large uncertainties $(>50 \%)$ in the intensities are to be expected, in particular when comparing lines not close in wavelength. The spectral accuracy and resolution $(\simeq 0.002 \AA$ on average) was excellent, and the spectra covered an extended wavelength range. The spectra were obtained using Nd-glass laser emission focused on flat target surfaces with pulses of half-width of $\simeq 2 \mathrm{~ns}$. The plasma observed had typical densities of the order of $10^{18}-10^{20} \mathrm{~cm}^{-3}$ and temperatures of a few $10^{7} \mathrm{~K}$. At such high densities, many lower levels become significantly populated, and many line ratios become highly density-sensitive.

After the early solar observations, further improvements in terms of spectral resolution was achieved with the SOLEX spectrometers (see McKenzie et al. 1980, 1985), although the data lacked accuracy in wavelengths and radiometric calibration. The Solar Maximum Mission (SMM) flat crystal spectrometers (FCS) data achieved a good spectral resolution. Phillips et al. (1982) reported SMM/FCS spectra in the 6-19 range, containing $n=3 \rightarrow 2$ lines. An SMM/FCS solar flare spectrum of excellent quality, with $n=4 \rightarrow 2$ calibrated line intensities was published by Fawcett et al. (1987). The main limitation of all the above solar observations was the fact that the spectra were recorded by scanning the wavelength range, hence different lines were not observed simultaneously. This can be a problem if the intensities vary considerably during a flare.

In recent years, new laboratory measurements have been published. Among them, a tokamak spectrum in the 7-9 $\AA$ region of excellent spectral resolution was provided by Wargelin et al. (1998). The authors gave line identifications but no line intensities. Recently, Electron Beam Ion Trap (EBIT) spectra containing the few brightest $n=3 \rightarrow 2$ lines have been published by Brown et al. (2002). Compared to laser spectra, the advantage of tokamak and EBIT spectra is the low density (similar to that one of solar flares), and the presence of lines only from a restricted range of ions. The limitations of these laboratory data are the poor spectral resolution, the low signalto-noise, the lack of a radiometric calibration and the complexities in modelling the plasma emission.

Further observations of Fe XXIV $n=3,4 \rightarrow 2$ spectral lines for a variety of "hot" astrophysical sources have been provided by the gratings on-board the Chandra and XMM-Newton satellites. They represent an important improvement over the best solar observations because the lines were simultaneously recorded. Note that the spectral resolution of the Chandra high-energy transmission grating (HETG) spectra is comparable to the best solar ones. A consistent radiometric calibration of all the different Chandra and XMM-Newton channels is still lacking, but the intensities can be considered accurate to within an acceptable $30 \%$ or so.

\section{The benchmark method}

The general procedures of the benchmark method are described in detail in Del Zanna et al. (2004), while specific issues related to laser spectra are discussed in Del Zanna et al. (2005). In summary, steady-state collisionally-ionised optically-thin emission in a plasma with electrons having a Maxwellian distribution is assumed. This is appropriate to model the majority of spectroscopic observations of solar and stellar coronae. Given the high electron density and the very short time-scales for the electron-electron collisions (compared to the other relevant processes), the assumptions of Maxwellian distributions and of steady-state plasma are reasonable even to model dynamic events such as solar flares (except perhaps the impulsive phase). In the case of laboratory plasmas, the validity of these assumptions depends on the particular experiment. For the laser spectra considered here, the lifetimes of the excited states were much shorter than the timescales over which the plasma conditions varied. Hence, the assumption of steady-state conditions is reasonable.

It is important to keep in mind, however, that the timescales for ionisation and recombination processes even at the high laser plasma densities are normally higher than the gasdynamic and pulse width timescales, and therefore the plasma is not in ionization equilibrium. In case of solar flares, there is contradicting evidence on the existence of departures from ionization equilibrium (see, e.g. the review of Doschek 1990).

The benchmark method is composed of an iterative procedure. Observed energy levels $E_{\text {obs }}$ (together with their uncertainties, based on the uncertainties in the observed wavelengths) are defined using the identified transitions (normally the brightest lines). Then, the "term energy correction" (TEC) procedure (see, e.g. Zeippen et al. 1977; Nussbaumer \& Storey 1978) within the atomic structure code SUPERSTRUCTURE (SS, see Eissner et al. 1974) is applied, to obtain empiricallyadjusted fine-structure energies $E_{\mathrm{SS}}$ and calculate the spontaneous transition probabilities $A_{j i}$. The $A_{j i}$ values are then benchmarked using measurements of lifetimes and branching ratios, whenever available. The $A_{j i}$ values, together with the collisional data, are then used to calculate the fractional population $N_{j}\left(N_{\mathrm{e}}, T_{\mathrm{e}}\right)$ of the upper level $j$ (relative to the total number density of the ion), as a function of electron temperature $T_{\mathrm{e}}$ and density $N_{\mathrm{e}}$. The theoretical intensities (proportional to $N_{j} A_{j i}$ ) at different density and temperature regimes are compared to the observed intensities $I_{\mathrm{ob}}$, by plotting the "emissivity ratio curves":

$F_{j i}\left(N_{\mathrm{e}}, T_{\mathrm{e}}\right)=C \frac{I_{\mathrm{ob}} N_{\mathrm{e}}}{N_{j}\left(N_{\mathrm{e}}, T_{\mathrm{e}}\right) A_{j i}}$

calculated at a fixed temperature $T_{\mathrm{e}}=T_{0}$ (or at a fixed density $N_{\mathrm{e}}=N_{0}$ ) as a function of the electron density $N_{\mathrm{e}}$ (or temperature $T_{\mathrm{e}}$ ). For the astrophysical spectra considered here, a fixed 
density $10^{12} \mathrm{~cm}^{-3}$, typical of solar flares, has been adopted. Note, however, that the emissivity ratios are virtually insensitive to densities up to $10^{17} \mathrm{~cm}^{-3}$. For the spectra from laser plasmas, a fixed temperature of $10^{7.3} \mathrm{~K}$ has been assumed. Variations of this temperature do not significantly affect the results presented here.

The proportionality constant $C$ is chosen for each dataset so that the emissivity ratios are close to unity. If agreement between theory and observations holds, the $F_{j i}$ values for different spectral lines should approximately overlap.

We use the emissivity ratio curves primarily to assess line identifications and blending. This method is equivalent to the widely-used line ratio one, but has the advantage of providing an overall view for all the spectral lines at once. The method and the model ion are quite simple, but at least have the advantage that excitations and radiative cascades are taken into account, and line intensities are used, instead of a simple use of $g f$ values. The line identifications are adjusted and the procedure is repeated in order to identify all the spectral lines that should be observable and provide a set of best energies $E_{\text {best }}$. These energies are the adjusted observed energies, whenever available, and the adjusted $E_{\mathrm{SS}}$ values otherwise.

The $F_{j i}$ curves can also be used as a diagnostic tool. Curves of spectral lines that have a different density (or temperature) dependence should approximately intersect around a value which is the average density (or temperature) of the emitting plasma. The average would be over the spatial region were the plasma is formed, and over time.

However, one should keep in mind that plasmas can be multi-thermal and multi-density, hence accurate measurements are difficult to obtain (this is a well-known problem with any remote-sensing measurement).

Here, the electron impact collision strengths calculated by Berrington \& Tully (1997) and Whiteford et al. (2002) (hereafter BT97 and W02 respectively) are used in the benchmark of Fe XXIV L-shell emission. They represent the most accurate $R$-matrix calculations available at this time. For a discussion of previous distorted-wave (DW) scattering calculations see BT97 and W02. BT97 performed a full Breit-Pauli (BP) $R$-matrix calculation by including all resonances up to $n=4$ for singlyexcited levels. W02 performed an intermediate-coupling frame transformation method (ICFT) $R$-matrix calculation which included singly excited levels up to $n=5$, and also inner-shell transitions up to $n=3$. The two methods (BP and ICFT) give similar results, although the BP one in theory should be more accurate. We have found that differences in the collision strengths are small (of the order of few \%) and normally at lower temperatures.

\section{Results}

Table 1 presents a summary of the observed energies (or adjusted $E_{\mathrm{SS}}$ values), compared to the energies available from the NIST database v. $3^{1}$ for the lowest 24 configurations. These are the configurations adopted to calculate the radiative data in intermediate coupling and including the fine-structure two-body

\footnotetext{
${ }^{1}$ http://physics.nist.gov/PhysRefData/ASD/index.html
}

Table 1. The details of the most important configurations in Fe XXIV. $E_{\text {best }}$ indicates the best energies $\left(\mathrm{cm}^{-1}\right)$ proposed here. The uncertainties in the energies reflect the estimated errors in the wavelength measurements. Levels with uncertain identification are assigned an uncertainty of $5000 \mathrm{~cm}^{-1}$. The following columns indicate the differences between our $E_{\text {best }}$ and the energies from NIST $E_{\mathrm{NIST}}$ (v.3), the collisional calculations $E_{\mathrm{CC}}$ of $\mathrm{W} 02$, and the adjusted SS values $E_{\mathrm{SS}}$.

\begin{tabular}{|c|c|c|c|c|c|c|}
\hline$i$ & $\begin{array}{l}\text { Conf. } \\
\left(1 s^{2}\right)\end{array}$ & Term & $E_{\text {best }}$ & $\begin{array}{l}E_{\text {best }^{-}} \\
E_{\text {NIST }}\end{array}$ & $\begin{array}{r}E_{\text {best }^{-}} \\
E_{\mathrm{CC}}\end{array}$ & $\begin{array}{l}E_{\text {best }} \\
-E_{\mathrm{SS}}\end{array}$ \\
\hline 1 & $2 \mathrm{~s}$ & $e_{1 / 2}^{e}$ & $0.0=$ & 0 & TU & 0 \\
\hline 2 & $2 p$ & ${ }^{2} \mathrm{P}_{1 / 2}^{\mathrm{o}}$ & $391983.0 \pm 8$ & 0 & -608 & -921 \\
\hline 3 & $2 p$ & ${ }^{2} \mathrm{P}_{3 / 2}^{\mathrm{o}}$ & $520757.0 \pm 14$ & 0 & +716 & +921 \\
\hline 4 & $3 \mathrm{~s}$ & ${ }^{2} \mathrm{~S}_{1 / 2}^{\mathrm{e} / 2}$ & $9271900.0 \pm 1000$ & -600 & -4333 & \\
\hline 5 & $3 p$ & ${ }^{2} \mathrm{P}_{1 / 2}^{\mathrm{o}}$ & $9378200.0 \pm 1000$ & 0 & -6501 & -1037 \\
\hline 6 & $3 p$ & ${ }^{2} \mathrm{P}_{3 / 2}^{\mathrm{o}}$ & $9417100.0 \pm 1000$ & 0 & -4675 & +1036 \\
\hline 7 & $3 \mathrm{~d}$ & ${ }^{2} \mathrm{D}_{3 / 2}^{\mathrm{e} / \mathrm{2}}$ & $9458990.0 \pm 1000$ & -10 & -6655 & -758 \\
\hline 8 & $3 d$ & ${ }^{2} \mathrm{D}_{5 / 2}^{\mathrm{e} / \mathrm{e}}$ & $9472500.0 \pm 2500$ & -100 & -5159 & +757 \\
\hline 9 & $4 \mathrm{~s}$ & ${ }^{2} \mathrm{~S}_{1 / 2}^{\mathrm{e}^{2}}$ & $12461000.0 \pm 3000$ & -3400 & -8633 & -2 \\
\hline 10 & $4 p$ & ${ }^{2} \mathrm{P}_{1 / 2}^{\mathrm{o} / 2}$ & $12510900.0 \pm 4000$ & 2000 & -3265 & +554 \\
\hline 11 & $4 p$ & ${ }^{2} \mathrm{P}_{3 / 2}^{\mathrm{o}}$ & $12525000.0 \pm 4000$ & 100 & -4499 & -551 \\
\hline 12 & $4 \mathrm{~d}$ & ${ }^{2} \mathrm{D}_{3 / 2}^{\mathrm{e} / \mathrm{z}}$ & $12538800.0 \pm 2000$ & -400 & -8876 & -688 \\
\hline 13 & $4 d$ & ${ }^{2} \mathrm{D}_{5 / 2}^{\mathrm{e} / 2}$ & $12545200.0 \pm 2700$ & 0 & -7512 & +688 \\
\hline 14 & $4 \mathrm{f}$ & ${ }^{2} \mathrm{~F}_{5 / 2}^{\mathrm{o}}$ & $12547600.0 \pm 2000$ & 0 & -6125 & -686 \\
\hline 15 & $4 \mathrm{f}$ & ${ }^{2} \mathrm{~F}_{7 / 2}^{\mathrm{o}}$ & $12551500.0 \pm 2000$ & -100 & -4754 & +686 \\
\hline 16 & $5 \mathrm{~s}$ & ${ }^{2} S_{1 / 2}^{e}$ & $13925450.0 \pm 5000$ & 0 & -9626 & -1 \\
\hline 17 & $5 p$ & ${ }^{2} \mathrm{P}_{1 / 2}^{\mathrm{o}}$ & $13941339.0 \pm 5000$ & -2061 & -16177 & \\
\hline 18 & $5 p$ & ${ }^{2} \mathrm{P}_{3 / 2}^{\mathrm{o}}$ & $13948900.0 \pm 4000$ & -2700 & -16261 & -1 \\
\hline 19 & $5 d$ & ${ }^{2} \mathrm{D}_{3 / 2}^{\mathrm{e} / \mathrm{e}}$ & $13960500.0 \pm 4000$ & 0 & -13891 & -1130 \\
\hline 20 & $5 d$ & ${ }^{2} \mathrm{D}_{5 / 2}^{\mathrm{e} / \mathrm{d}}$ & $13965300.0 \pm 4000$ & 0 & -11640 & +1130 \\
\hline 21 & $5 f$ & ${ }^{2} \mathrm{~F}_{5 / 2}^{\mathrm{o} / 2}$ & $13964041.0 \pm 5000$ & - & -13469 & (2) \\
\hline 22 & $5 f$ & ${ }^{2} \mathrm{~F}_{7 / 2}^{\mathrm{O}}$ & $13965328.0 \pm 5000$ & - & -13470 & \\
\hline 23 & $5 \mathrm{~g}$ & ${ }^{2} \mathrm{G}_{7 / 2}^{\mathrm{e}}$ & $13965337.0 \pm 5000$ & - & -13470 & ( \\
\hline 24 & $5 \mathrm{~g}$ & ${ }^{2} \mathrm{G}_{9 / 2}^{\mathrm{e}}$ & $13966112.0 \pm 5000$ & - & -13470 & 0 \\
\hline
\end{tabular}

interactions. For a simple ion such as Fe XXIV the agreement between observed energies and those calculated adopting different approaches or structure codes are very similar, as one would expect. We note good agreement (mostly within uncertainties) between the observed energies and those recently released with the NIST database v.3, which is largely based on the compilation of Shirai et al. (2000).

The $A$ values have been calculated with SUPERSTRUCTURE and using the best energies. These values, as expected, compare well (within 10\%) with those available in the literature. The calculated lifetimes of the $2 \mathrm{p}{ }^{2} \mathrm{P}_{3 / 2,1 / 2}$ levels are 231 and 548 ps respectively, which agree, within the experimental uncertainties, with the laboratory measurements (e.g., Dietrich et al. 1978 measured 235 \pm 10 and $555 \pm 20 \mathrm{ps}$ ). The $A$ values have been complemented with values for inner-shell transitions taken from W02.

The stationary level balance equations have been solved using the effective collision strengths of Berrington \& Tully (1997) for excitations up to $n=4$ and those of Whiteford et al. (2002) for the $n=5$ and inner-shell levels.

Table 2 shows the fractional level populations $N_{j}$ of the most important levels. It can be seen that, even at laser plasma 
Table 2. The fractional level populations $N_{j}$, calculated at different electron densities $\left(\mathrm{cm}^{-3}\right)$ and a temperature $\log T[\mathrm{~K}]=7$ for the lower $(n=2,3,4)$ levels. Values in brackets are obtained with the W02 data.

\begin{tabular}{ccccc}
\hline \hline$i$ & level & $10^{8}$ & $10^{14}$ & $10^{19}$ \\
\hline 1 & $2 \mathrm{~s}^{2} \mathrm{~S}_{1 / 2}$ & 1.00000 & 0.99997 & 0.43472 \\
2 & $2 \mathrm{p}^{2} \mathrm{P}_{1 / 2}$ & $1.5[1.6] \times 10^{-11}$ & $1.5 \times 10^{-5}$ & 0.24285 \\
3 & $2 \mathrm{p}^{2} \mathrm{P}_{3 / 2}$ & $1.2[1.3] \times 10^{-11}$ & $1.2 \times 10^{-5}$ & 0.32239 \\
4 & $3 \mathrm{~s}^{2} \mathrm{~S}_{1 / 2}$ & $2.1[2.1] \times 10^{-16}$ & $2.1 \times 10^{-10}$ & $1.1 \times 10^{-5}$ \\
5 & $3 \mathrm{p}^{2} \mathrm{P}_{1 / 2}$ & $3.4[3.6] \times 10^{-17}$ & $3.4 \times 10^{-11}$ & $4.1 \times 10^{-6}$ \\
6 & $3 \mathrm{p}^{2} \mathrm{P}_{3 / 2}$ & $6.6[7.0] \times 10^{-17}$ & $6.6 \times 10^{-11}$ & $7.1 \times 10^{-6}$ \\
7 & $3 \mathrm{~d}^{2} \mathrm{D}_{3 / 2}$ & $2.2[2.3] \times 10^{-17}$ & $2.2 \times 10^{-11}$ & $4.4 \times 10^{-6}$ \\
8 & $3 \mathrm{~d}^{2} \mathrm{D}_{5 / 2}$ & $3.3[3.4] \times 10^{-17}$ & $3.3 \times 10^{-11}$ & $5.5 \times 10^{-6}$ \\
9 & $4 \mathrm{~s}^{2} \mathrm{~S}_{1 / 2}$ & $3.6[4.1] \times 10^{-17}$ & $3.6 \times 10^{-11}$ & $1.8 \times 10^{-6}$ \\
10 & $4 \mathrm{p}^{2} \mathrm{P}_{1 / 2}$ & $8.1[9.1] \times 10^{-18}$ & $8.1 \times 10^{-12}$ & $8.9 \times 10^{-7}$ \\
11 & $4 \mathrm{p}^{2} \mathrm{P}_{3 / 2}$ & $1.6[1.8] \times 10^{-17}$ & $1.6 \times 10^{-11}$ & $1.6 \times 10^{-6}$ \\
12 & $4 \mathrm{~d}^{2} \mathrm{D}_{3 / 2}$ & $5.5[5.8] \times 10^{-18}$ & $5.5 \times 10^{-12}$ & $1.2 \times 10^{-6}$ \\
13 & $4 \mathrm{~d}^{2} \mathrm{D}_{5 / 2}$ & $8.3[8.7] \times 10^{-18}$ & $8.3 \times 10^{-12}$ & $1.5 \times 10^{-6}$ \\
14 & $4 \mathrm{f}^{2} \mathrm{~F}_{5 / 2}$ & $5.8[6.8] \times 10^{-18}$ & $5.8 \times 10^{-12}$ & $6.8 \times 10^{-7}$ \\
15 & $4 \mathrm{f}^{2} \mathrm{~F}_{7 / 2}$ & $7.8[9.1] \times 10^{-18}$ & $7.8 \times 10^{-12}$ & $8.4 \times 10^{-7}$ \\
\hline & & & &
\end{tabular}

densities $\left(10^{19} \mathrm{~cm}^{-3}\right)$, only the ground and the $1 \mathrm{~s}^{2} 2 \mathrm{p}$ levels are significantly populated. BT97 provided collision strengths for excitations from the ground and the $1 s^{2} 2 p$ levels, which are the dominant contributors to the populations of the higher levels. Various tests have been performed, using different collision strengths or $A$-values. The result is that only small differences in the level populations are observed. For example, Table 2 shows in brackets the populations calculated with the W02 data in the low-density limit $\left(10^{8} \mathrm{~cm}^{-3}\right)$. Differences with the values calculated here are small, within $10 \%$, i.e. below the current experimental uncertainties.

Table 3 provides a summary list of all the lines that are predicted to be brightest at low and high densities, with a list of identifications. The second and third columns give the relative intensities of the brightest lines calculated at $10^{12}, 10^{19} \mathrm{~cm}^{-3}$, i.e. in the low-density limit (astrophysical plasmas) and at the densities of laser plasmas. The fourth column lists the wavelengths calculated from the best energies $E_{\text {best }}$, while the fifth column lists the observed wavelengths $\lambda_{\text {obs }}$. The last column indicates some of the original identifications found in the literature [note that clear assignments for original identifications are difficult to assess because many lines have often tentatively identified by various authors].

One positive result is that all the brightest lines in the two different regimes have been observed. All previous identifications are confirmed, although many lines turn out to be blended. All the lines observed in astrophysical plasmas have also been observed in laboratory, hence normally laboratory measurements are adopted here. We note that many laboratory wavelengths have been found to be inconsistent (i.e. not within the stated experimental uncertainties), so further refinements are still necessary. Some inconsistencies are noted in the table.

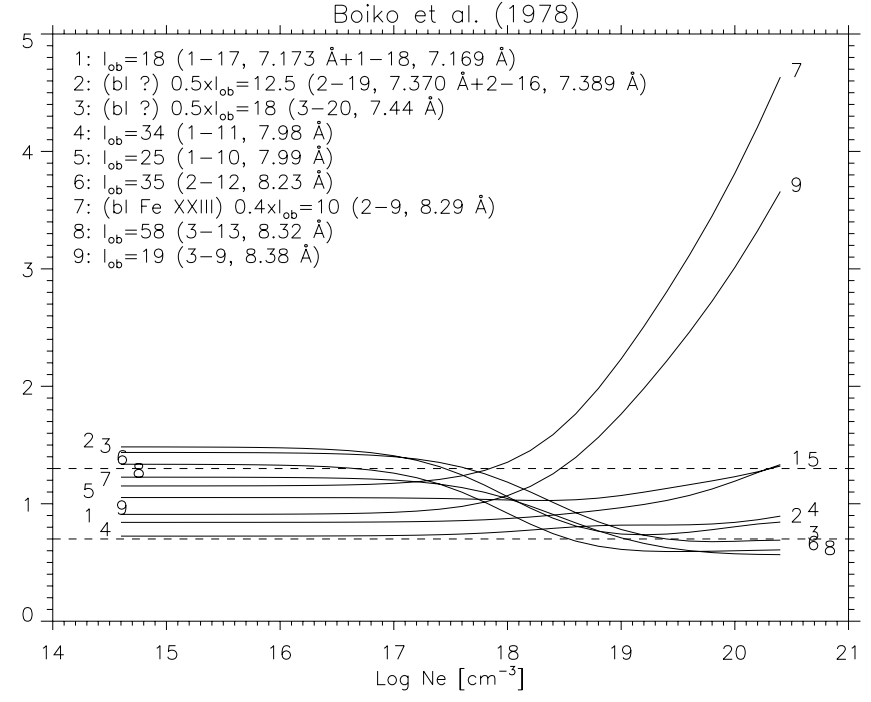

Fig. 1. The emissivity ratio curves relative to the $n=4,5 \rightarrow 2$ transitions observed in laser spectra by Boiko et al. (1978). The curves are labelled with increasing numbers. For each line, we indicate: the observed intensities $I_{\mathrm{ob}}$; the lower and upper level index corresponding to Table 1; the theoretical wavelength. Note that lines 2, 3, 7 appear to be strongly blended. The observed intensities of the blended lines have been reduced as indicated. For example, in line 7 only 0.4 of the measured intensity (totalling to 10 counts) is considered to be due to Fe XXIV, the rest being due to a known Fe XXIII transition. The curves show agreement within 30\% (dashed lines) and densities $\log N_{\mathrm{e}}\left[\mathrm{cm}^{-3}\right] \simeq 18$.

\section{1. $n=2 \rightarrow 2$ transitions}

$n=2 \rightarrow 2$ transitions fall in the EUV spectral range. They are important because accurate measurements of their wavelengths constrain the energies of the two $1 s^{2} 2 p$ levels. Neupert (1971) was the first to suggest the identification of the two strong 192, $255 \AA$ lines, from a solar flare spectrum. These lines are also dominant in spectra of flare stars (see, e.g. the EUVE spectra, Monsignori Fossi et al. 1996). For the wavelengths, the laboratory measurements of Reader et al. (1994) are adopted.

\section{2. $n=4,5 \rightarrow 2$ transitions}

$n=4,5 \rightarrow 2$ transitions provide good diagnostics to measure electron densities in laser plasmas, and, to a lesser extent, electron temperatures in tokamak or astrophysical sources. Figure 1 shows the emissivity ratio curves relative to the main $n=$ $4,5 \rightarrow 2$ transitions observed in the laser spectra by Boiko et al. (1978). The agreement between theory and observations is very good (within 30\%), when the large uncertainties in the line intensities are considered. The lines displayed in Fig. 1 are excellent density diagnostics at laser plasma temperatures. They consistently indicate $\log N_{\mathrm{e}} \simeq 18 \mathrm{~cm}^{-3}$, in excellent agreement with the results obtained from Fe XXIII (Del Zanna et al. 2005).

The same lines are also bright in astrophysical "hot" plasmas, such as those in solar flares. Figure 2 shows the emissivity ratio curves relative to the 1969 February 27 solar flare recorded with the KAP crystal spectrometer on-board OSO-5 (Neupert et al. 1973). The spectra were radiometrically 
Table 3. Summary of the line identifications for Fe XXIV The columns indicate: 1) the indexes corresponding to Table 1;2) the relative intensity (in photons, at $10^{12}, 10^{19} \mathrm{~cm}^{-3}$ ), scaled to the 3-8 $11.171 \AA$ line; 3) the wavelengths calculated from the best energies $E_{\text {best }}$; 4) observed wavelengths $\lambda_{\mathrm{obs}}$; some blends are indicated ( $\mathrm{bl}=$ blend; $\mathrm{bl}-\mathrm{mr}=$ blend in medium-resolution spectra; $\mathrm{bl}-\mathrm{w}=\mathrm{blend}$ with a weak line); lines with no or a tentative identification have a question mark; 5) some of the previous original identifications. Bo78: Boiko et al. (1978); B77: Bromage et al. (1977); D72: Doschek et al. (1972); F79: Fawcett et al. (1979); N71:Neupert (1971); N73: Neupert et al. (1973); R94: Reader et al. (1994); SF86: Seely \& Feldman (1986); W98: Wargelin et al. (1998).

\begin{tabular}{|c|c|c|c|c|c|}
\hline$i-j$ & $\begin{array}{c}\text { Int } \\
10^{12} \\
\end{array}$ & $\begin{array}{c}\text { Int } \\
10^{19}\end{array}$ & $\lambda_{\text {best }}(\AA)$ & $\lambda_{\mathrm{obs}}(\AA)$ & ID \\
\hline $1-18$ & $2.9 \times 10^{-2}$ & $2.5 \times 10^{-2}$ & $7.169 \pm 0.002$ & $7.169 \pm 0.002$ Bo78,W02 (bl Al XIII ?) & Bo78 \\
\hline $1-17$ & $1.4 \times 10^{-2}$ & $1.3 \times 10^{-2}$ & $7.173 \pm 0.003$ & (bl 7.169) & Bo78 \\
\hline $2-19$ & $1.4 \times 10^{-2}$ & $3.3 \times 10^{-2}$ & $7.370 \pm 0.002$ & $7.370 \pm 0.002$ Bo78, W02 (bl ?) & N73 \\
\hline $3-20$ & $2.5 \times 10^{-2}$ & $4.7 \times 10^{-2}$ & $7.438 \pm 0.002$ & $7.438 \pm 0.002$ Bo78 (bl ?) & N73 \\
\hline $3-19$ & $2.8 \times 10^{-3}$ & $6.6 \times 10^{-3}$ & $7.441 \pm 0.002$ & weak (bl 7.445 Bo78?) & \\
\hline $3-16$ & $1.1 \times 10^{-2}$ & $4.7 \times 10^{-3}$ & $7.460 \pm 0.003$ & $7.457 \pm 0.001 \mathrm{~W} 02$ & ? W02 \\
\hline $1-11$ & $9.5 \times 10^{-2}$ & $8.4 \times 10^{-2}$ & $7.984 \pm 0.003$ & $7.984 \pm 0.001$ F79 (W02 7.9857 ?) & D72 \\
\hline $1-10$ & $4.8 \times 10^{-2}$ & $4.7 \times 10^{-2}$ & $7.993 \pm 0.003$ & $7.993 \pm 0.002$ Bo78 (W02 $7.9960 ?)$ & Bo78 \\
\hline $2-12$ & $5.3 \times 10^{-2}$ & 0.12 & $8.233 \pm 0.001$ & $8.2326 \pm 0.0007$ W02 (Bo78 8.231) & D72 \\
\hline $2-9$ & $1.8 \times 10^{-2}$ & $9.1 \times 10^{-3}$ & $8.286 \pm 0.002$ & $8.2854 \pm 0.0007$ SF86 (bl Fe XXIII) & D72 \\
\hline $3-13$ & $9.6 \times 10^{-2}$ & 0.17 & $8.316 \pm 0.002$ & $8.3160 \pm 0.0007$ SF86 (bl Fe XXIII) & D72 \\
\hline $3-12$ & $1.0 \times 10^{-2}$ & $2.3 \times 10^{-2}$ & $8.321 \pm 0.001$ & 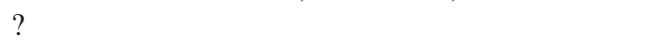 & \\
\hline $3-9$ & $4.2 \times 10^{-2}$ & $2.2 \times 10^{-2}$ & $8.375 \pm 0.002$ & $8.3757 \pm 0.0007$ SF86 (bl unid.) & D72 \\
\hline $1-6$ & 0.76 & 0.70 & $10.619 \pm 0.001$ & $10.619 \pm 0.002$ Bo78 $(10.620$ F79, bl Fe XIX B77) & N73 \\
\hline $1-5$ & 0.40 & 0.42 & $10.663 \pm 0.001$ & $10.663 \pm 0.002$ Bo78 $(10.662$ F79, bl Fe XIX B77) & N73 \\
\hline $2-7$ & 0.55 & 1.1 & $11.029 \pm 0.001$ & $11.030 \pm 0.002$ Bo78 (bl mr Fe XXIII 11.018) & N73 \\
\hline $3-8$ & 1.0 & 1.6 & $11.171 \pm 0.003$ & $11.171 \pm 0.003$ Bo78 (11.174 F79) & N73 \\
\hline $3-7$ & 0.11 & 0.22 & $11.188 \pm 0.001$ & $11.187 \pm 0.002$ Bo78 (bl Na X ? - F79 11.186) & Bo78 \\
\hline $2-4$ & 0.23 & 0.13 & $11.261 \pm 0.001$ & $11.261 \pm 0.002$ Bo78 (bl Fe XVII,XVIII B77) & Bo78 \\
\hline $3-4$ & 0.51 & 0.28 & $11.427 \pm 0.001$ & $11.426 \pm 0.002$ Bo78 (bl Fe XVIII B77, Fe XXIII) & Bo78 \\
\hline $7-21$ & $1.2 \times 10^{-2}$ & $1.4 \times 10^{-2}$ & $22.197 \pm 0.030$ & - & \\
\hline $8-22$ & $1.7 \times 10^{-2}$ & $1.7 \times 10^{-2}$ & $22.258 \pm 0.037$ & - & \\
\hline $4-11$ & $2.9 \times 10^{-2}$ & $2.6 \times 10^{-2}$ & $30.740 \pm 0.047$ & $30.743 \pm 0.004 \mathrm{R} 94$ & R94 \\
\hline $4-10$ & $1.5 \times 10^{-2}$ & $1.5 \times 10^{-2}$ & $30.874 \pm 0.048$ & $30.895 \pm 0.004$ R94 & R94 \\
\hline $5-12$ & $1.7 \times 10^{-2}$ & $3.8 \times 10^{-2}$ & $31.640 \pm 0.030$ & $31.637 \pm 0.004$ R94 & R94 \\
\hline $6-13$ & $3.2 \times 10^{-2}$ & $5.5 \times 10^{-2}$ & $31.968 \pm 0.038$ & $31.968 \pm 0.004 \mathrm{R} 94$ & R94 \\
\hline $7-14$ & $3.9 \times 10^{-2}$ & $4.7 \times 10^{-2}$ & $32.377 \pm 0.031$ & $32.377 \pm 0.002 \mathrm{R} 94$ & R94 \\
\hline $8-15$ & $5.6 \times 10^{-2}$ & $5.9 \times 10^{-2}$ & $32.478 \pm 0.047$ & $32.478 \pm 0.002 \mathrm{R} 94$ & R94 \\
\hline $6-9$ & $3.2 \times 10^{-2}$ & $1.6 \times 10^{-2}$ & $32.853 \pm 0.043$ & - & \\
\hline $1-3$ & 40. & 12. & $192.028 \pm 0.005$ & $192.028 \pm 0.005$ R94 & N71 \\
\hline $1-2$ & 21. & 4.0 & $255.113 \pm 0.005$ & $255.113 \pm 0.005 \mathrm{R} 94$ & N71 \\
\hline
\end{tabular}

calibrated, and were of excellent quality, although the wavelength calibration was not very good (cf. Fig. 2). Note that many lines in the original Neupert et al. (1973) line list were still not identified at the time. Agreement between observed and calculated intensities is reasonably good (within 50\%), considering that the intensities were averaged over various spectra taken at different times during the flare.

Figure 3 shows the emissivity ratio curves relative to the $n=4 \rightarrow 2$ transitions observed with the SOLEX A spectrometer during the 1981 May 5 flare and measured by McKenzie et al. (1985). The lines were observed within less than a minute, and the variations in flare line intensities were less than $25 \%$ (McKenzie et al. 1985). The agreement between theory and observations is good, within $30 \%$.

Figure 4 shows the emissivity ratio curves relative to the $n=4 \rightarrow 2$ transitions observed by the SMM/FCS during the 1985 July 2 solar flare and measured by Fawcett et al. (1987). The Fe XXIV lines were observed just after the peak of the flare and to within a few minutes, during which intensities of the flare lines decreased by at most 10\% (see Del Zanna et al. 2005). The lines can therefore be considered as observed simultaneously. The curves show excellent agreement between theory and observations (within 20\%), and are consistent with the plasma being isothermal with $\log T \simeq 7.2-7.4$. Note that for the $8.317 \AA$ line, a $30 \%$ contribution due to Fe XXIII (see Del Zanna et al. 2005) has been removed.

Now a few more comments on individual lines are worth giving. The $7.169 \AA$ line is in good agreement in the laboratory laser spectra. In astrophysical spectra, this line is often attributed entirely to Al XIII (as done by McKenzie et al. 1985) and not to Fe XXIV. It appears, however, that blending occurs. The 7.370, 7.438 $\AA$ lines are blended in the laser spectra, but not in the solar flare spectrum of Neupert et al. (1973). The 7.984, 7.993, 8.233 $\AA$ lines are in excellent agreement in both laboratory and solar spectra, indicating that they are not blended (in the highest-resolution spectra). The $8.285 \AA$ 


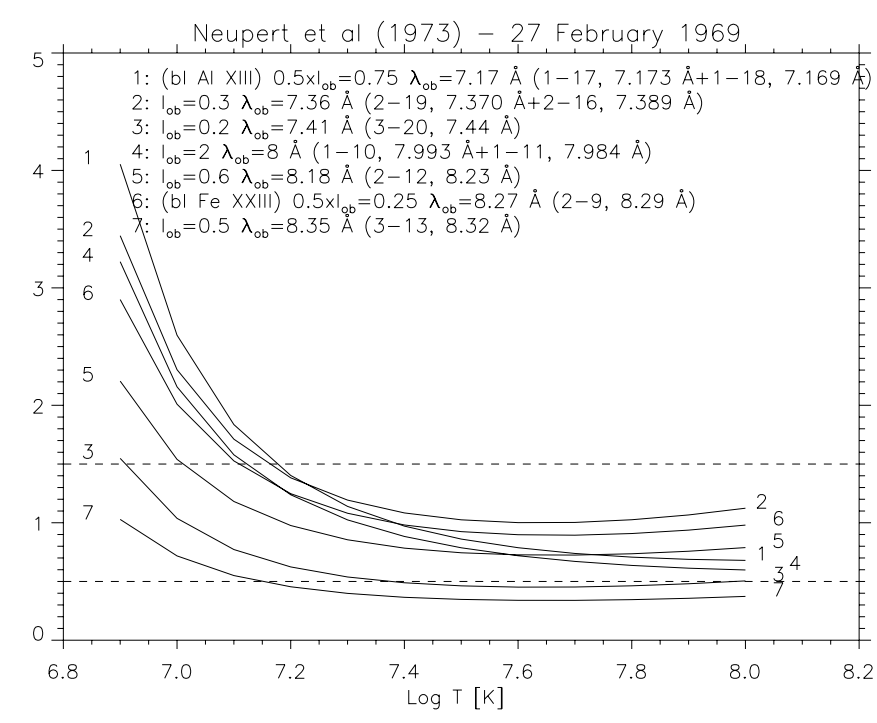

Fig. 2. The emissivity ratio curves relative to the $n=4 \rightarrow 2$ transitions observed in the 1969 February 27 solar flare (Neupert et al. 1973). The observed wavelengths $\lambda_{\mathrm{ob}}$ are also indicated.

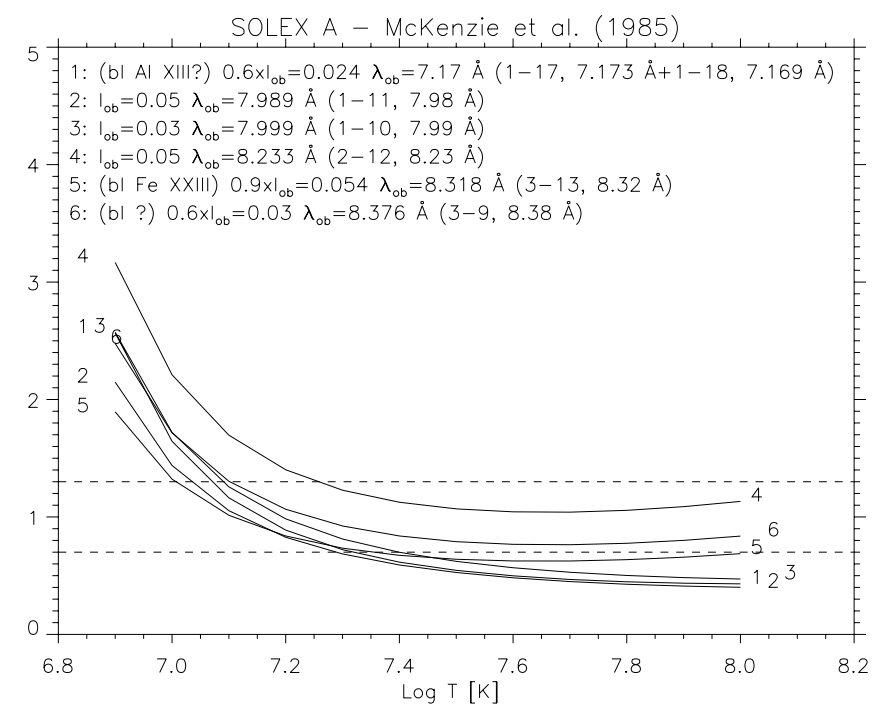

Fig. 3. The emissivity ratio curves relative to the $n=4 \rightarrow 2$ transitions observed with the SOLEX A spectrometer (McKenzie et al. 1985).

line is weaker and blended with Fe XXIII. The $8.316 \AA$ line is a stronger line, that in all spectra is blended with a weaker Fe XXIII 8.315 A line (see Del Zanna et al. 2005). In mediumresolution spectra, this line is further blended with a strong Fe XXIII 8.303 $\AA$ line. Finally, the $8.376 \AA$ line is weak and appear not blended in the laser spectra. In solar flare spectra, this line is obviously blended. Indeed there are at least two unidentified lines longward of this transition, one of which was measured at $8.405 \AA$ by Seely \& Feldman (1986) in their excellent solar flare spectrum.

\section{3. $n=3 \rightarrow 2$ transitions}

Fe XXIV $n=3 \rightarrow 2$ transitions fall in the 10-12 $\AA$ range. The number of Fe XXIV spectral lines observed in astrophysical plasmas is very small. The same lines are relatively strong

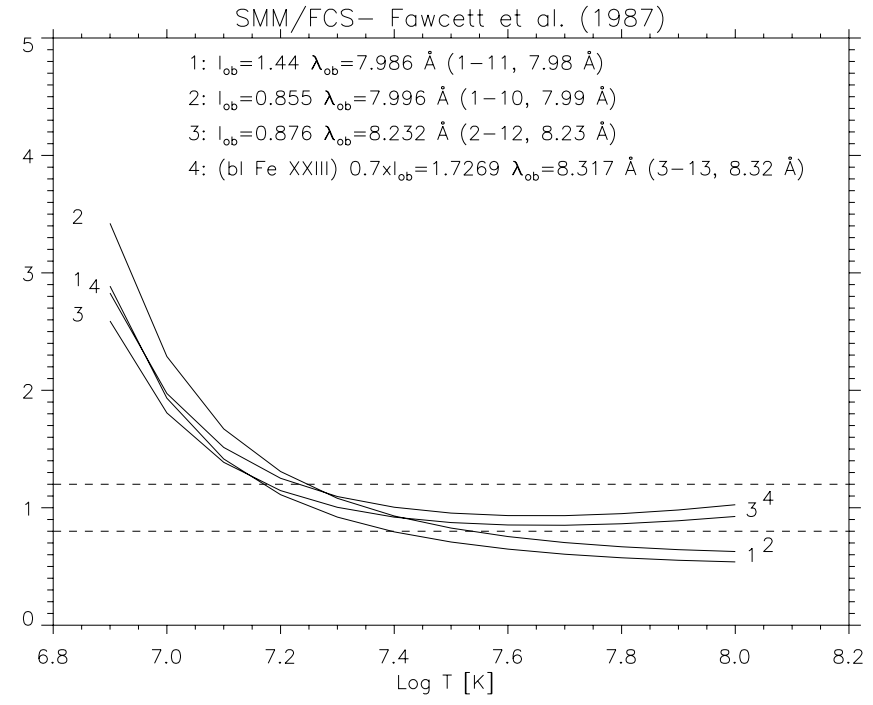

Fig. 4. The emissivity ratio curves relative to the $n=4 \rightarrow 2$ transitions observed in the SMM/FCS solar flare spectra by Fawcett et al. (1987).

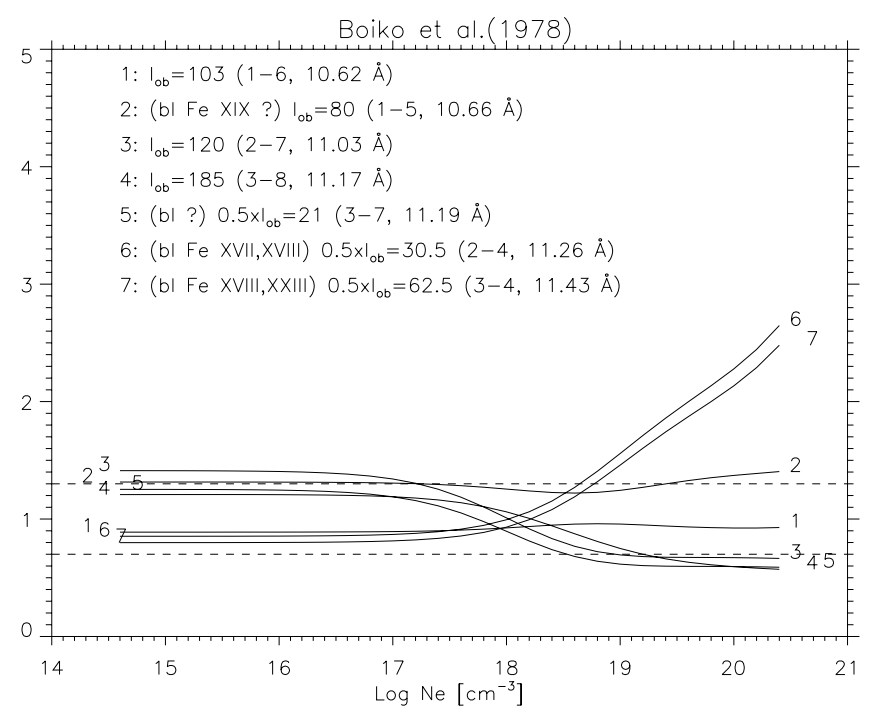

Fig. 5. The $F_{j i}$ curves relative to the $n=3 \rightarrow 2$ transitions observed in laser spectra by Boiko et al. (1978).

both at astrophysical and laser plasma densities, but are often blended with transitions from lower ionization stages. There is a large body of literature on astrophysical and laboratory observations of $n=3 \rightarrow 2$ transitions, with many uncertain or contradicting identifications, in particular for Fe XVII, Fe XVIII, Fe XIX, which emit a host of lines in the 10-12 A range that often blend Fe XXIV lines. Blending is often unique for each observed spectrum, so it is useful to assess which lines can be considered basically free of blends. To help the identification process, use has been made of the excellent laboratory spectra of Bromage et al. (1977), where iron emission due to ionization stages higher than XXI was not present.

Figure 5 shows the $F_{j i}$ curves relative to the brightest $n=3 \rightarrow 2$ transitions observed in the laser spectra by Boiko et al. (1978). The agreement between theory and observations is very good (within $30 \%$ ) also in this case, indicating the same electron densities as obtained from the $n=4 \rightarrow 2$ transitions. 


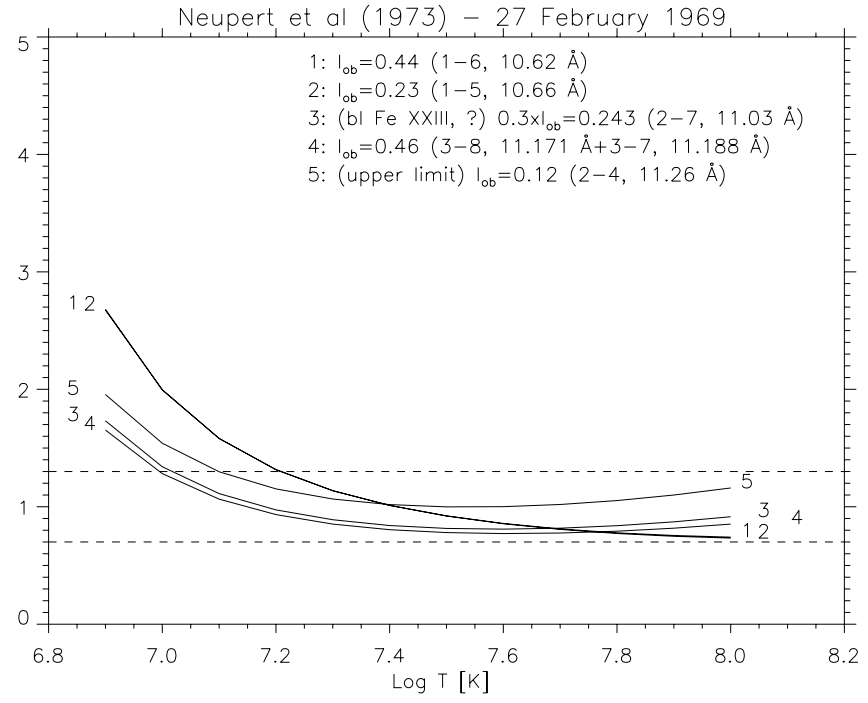

Fig. 6. The emissivity ratio curves relative to the $n=3 \rightarrow 2$ transitions observed in the 1969 February 27 solar flare (Neupert et al. 1973).

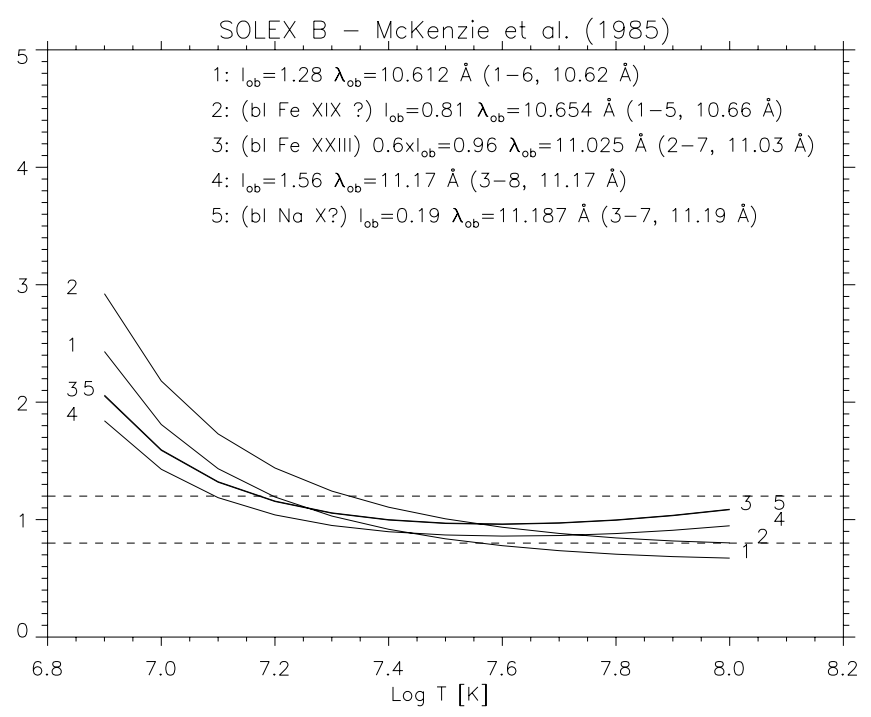

Fig. 7. The $F_{j i}$ curves relative to the $n=3 \rightarrow 2$ transitions observed with the SOLEX B spectrometer (McKenzie et al. 1985).

Figure 6 shows the emissivity ratio curves for the line intensities of the 1969 February 27 solar flare recorded with the KAP crystal spectrometer on-board OSO-5 and measured by Neupert et al. (1973). The 10.61, $10.65 \AA$ lines were separated in second order. Good (within 30\%) agreement is found.

Figure 7 shows the emissivity ratio curves relative to the $n=3 \rightarrow 2$ transitions observed with the SOLEX B spectrometer (McKenzie et al. 1985). The agreement between theory and observations is very good, within $20 \%$.

Now a few comments on some individual lines. In general, the $10.619,10.663 \AA$ lines are both blended with Fe XIX transitions. Whenever Fe XXIV emission is strong, the $10.619 \AA$ line appears free of blending, while the $10.663 \AA$ line is only partially blended with Fe XIX.

The $11.030 \AA$ is a strong line, that in the high-resolution laser spectra appears not blended. This line is in a spectral region crowded with lines, some of which have not been

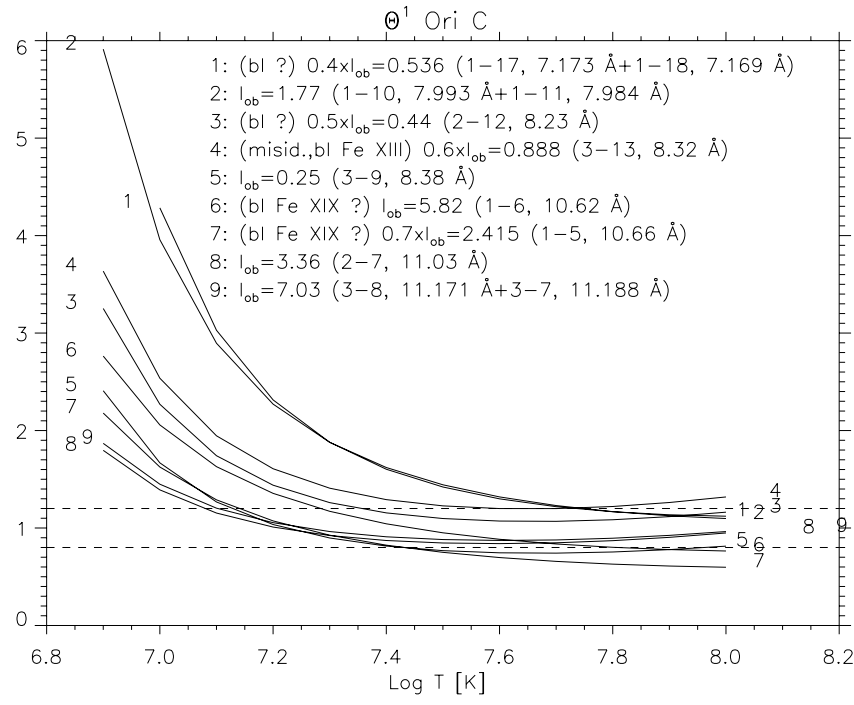

Fig. 8. The $F_{j i}$ curves relative to the $n=3,4 \rightarrow 2$ transitions observed with the Chandra gratings. Note that the combination of $n=3,4 \rightarrow 2$ transitions could be a good temperature diagnostic, but that the $n=$ $4 \rightarrow 2$ appear to be over-estimated.

identified yet. The brightest identified line is the Fe XXIII $11.018 \AA$ (see Del Zanna et al. 2005), and indeed in most solar spectra these lines are blended. The $11.171 \AA$ line is the strongest Fe XXIV transition in the group, often blended with the weaker Fe XXIV 11.187 $\AA$ line. Note that the $11.187 \AA$ line appears to be blended in the laser spectra of Boiko et al. (1978). However, in the high-resolution SOLEX spectra the observed line appears to be due solely to Fe XXIV (and not to Na X as suggested by McKenzie et al. 1985). Finally, the 11.261, $11.426 \AA$ lines are strongly blended with bright lower-temperature ions. In summary, unless the flare lines are dominant, most of the Fe XXIV lines are considerably blended with lower-temperature ions, with the exception of the 10.618 and $11.171 \AA$ lines.

\subsection{Combined $n=2,3,4 \rightarrow 2$ transitions}

The spectral range of the Chandra HETG/MEG instruments allows for a simultaneous recording of the Fe XXIV $n=3,4 \rightarrow 2$ transitions, which could provide some useful temperature diagnostics. We found however that in many published papers the observed intensities of the $n=4 \rightarrow 2$ transitions are larger than expected, an indication of a possible calibration problem. As an example we show in Fig. 8 the emissivity ratio curves for the Chandra HETG observation reported by Schulz et al. (2003) of the young star $\Theta^{1}$ Ori $C$, the hottest in the Trapezium. The $n=3 \rightarrow 2$ lines are instead consistent (within 20\%) with an isothermal plasma at a temperature $\log T \simeq 7.3 \mathrm{~K}$, the same obtained from Fe XXIII (see Del Zanna et al. 2005), and in broad agreement with the value $(\log T \simeq 7.4 \mathrm{~K})$ of one of the peaks in the emission measure distribution calculated by Schulz et al. (2003). We note that Schulz et al. (2003) incorrectly identified the line observed at $8.304 \AA$ (due to Fe XXIII) with an Fe XXIV transition. On the contrary, the $8.316 \AA$ line was mistakenly assigned to Fe XXIII instead that to Fe XXIV. 


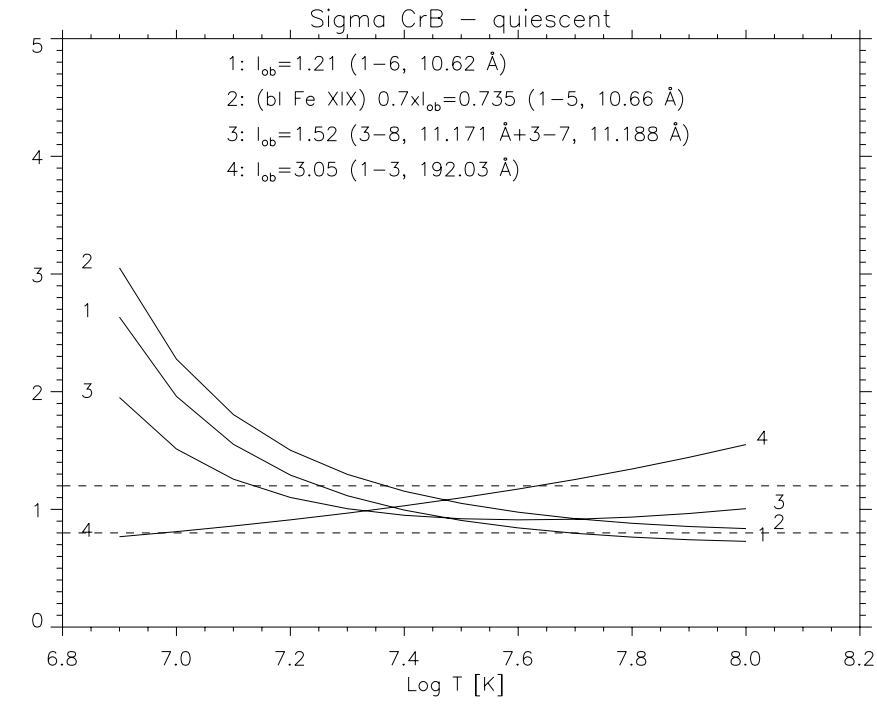

Fig. 9. The $F_{j i}$ curves relative to the $n=3 \rightarrow 2, n=2 \rightarrow 2$ transitions observed in the Chandra MEG and EUVE spectra of $\sigma^{2} \mathrm{CrB}$ during its quiescent state reported by Osten et al. (2003).

RS CVn stars produce X-ray spectra quite similar to those of solar flares and with strong Fe XXIV and Fe XXIII lines. Osten et al. (2003) reported a near-simultaneous Chandra and EUVE observation of the $\sigma^{2}$ CrB binary. The strong $n=2 \rightarrow 2192 \AA$ resonance line was observed by the EUVE spectrometers and its intensity corrected for interstellar $\mathrm{H}, \mathrm{He}$ absorption by Osten et al. (2003). For most of the observation the stars were in quiescence, hence even though the Chandra and EUVE observations were not strictly simultaneous, the line ratios should be approximately constant. Figure 9 shows the emissivity ratio curves relative to the Fe XXIV lines observed by Chandra and EUVE. The curves show good agreement (within 20\%), and are consistent with the coronae having an approximately isothermal temperature of $\log T[\mathrm{~K}] \simeq 7.3$, only marginally consistent with the emission measure distributions obtained by Osten et al. (2003), but in good agreement with the temperature $(\log T \simeq 7.3 \pm 0.1 \mathrm{~K})$ obtained with the Fe XXIII lines (Del Zanna et al. 2005). Note the blend of the $10.66 \AA$ with Fe XIX.

\section{Summary and conclusions}

Recent $R$-matrix calculations have been supplemented with radiative data and used to benchmark Fe XXIV L-shell emission against experimental data. Considering the large experimental uncertainties, we found good agreement in terms of wavelengths, line intensities and level lifetimes, thus giving confidence in the use of these atomic data. Most of the previous line identifications found in the literature have been confirmed on a quantitative basis. However, some identifications have been revised by taking into account the presence of blends. We have shown that Fe XXIV L-shell lines can be used to measure electron densities in laboratory plasmas and temperatures for a wide range of "hot" astrophysical sources. In particular, $n=3,4 \rightarrow 2$ transitions are an excellent density diagnostic in laser plasmas. $n=3,4 \rightarrow 2$ transitions can be used as temperature diagnostic for solar flare plasmas or any "hot" stellar corona, but well-calibrated and high-resolution measurements are required. An even better temperature diagnostic is the combination of $n=3,4 \rightarrow 2$ with $n=2 \rightarrow 2$ transitions, as shown in the case of the Chandra and EUVE observations of $\sigma^{2} \mathrm{CrB}$.

Acknowledgements. Support from PPARC is acknowledged.

\section{References}

Berrington, K. A., \& Tully, J. A. 1997, A\&AS, 126, 105

Boiko, V. A., Faenov, A. I., \& Pikuz, S. A. 1978, J. Quant. Spectr. Rad. Transfer., 19, 11

Bromage, G. E., Cowan, R. D., Fawcett, B. C., et al. 1977, RAL report, 170

Brown, G. V., Beiersdorfer, P., Liedahl, D. A., et al. 2002, ApJS, 140, 589

Chidichimo, M. C., Del Zanna, G., Mason, H. E., et al. 2005, A\&A, 430, 331

Del Zanna, G., Berrington, K. A., \& Mason, H. E. 2004, A\&A, 422, 731

Del Zanna, G., Chidichimo, M. C., \& Mason, H. E. 2005, A\&A, 432, 1137

Dietrich, D. D., Leavitt, J. A., Bashkin, S., et al. 1978, Phys. Rev. A, 18,208

Doschek, G. A. 1990, ApJS, 73, 117

Doschek, G. A., Meekins, J. F., \& Cowan, R. D. 1972, ApJ, 177, 261

Doschek, G. A., Meekins, J. F., \& Cowan, R. D. 1973, Sol. Phys., 29, 125

Eissner, W., Jones, M., \& Nussbaumer, H. 1974, Comput. Phys. Commun., 8, 270

Fawcett, B. C., Jordan, C., Lemen, J. R., \& Phillips, K. J. H. 1987, MNRAS, 225, 1013

Fawcett, B. C., Ridgeley, A., \& Hughes, T. P. 1979, MNRAS, 188, 365

McKenzie, D. L., Landecker, P. B., Broussard, R. M., et al. 1980, ApJ, 241, 409

McKenzie, D. L., Landecker, P. B., Feldman, U., \& Doschek, G. A. 1985, ApJ, 289, 849

Monsignori Fossi, B. C., Landini, M., Del Zanna, G., \& Bowyer, S. 1996, ApJ, 466, 427

Neupert, W. M. 1971, Phil. Trans. Roy. Soc. Lon. A, 270, 143

Neupert, W. M., Gates, W., Swartz, M., \& Young, R. 1967, ApJ, 149, L79

Neupert, W. M., Swartz, M., \& Kastner, S. O. 1973, Sol. Phys., 31, 171

Nussbaumer, H., \& Storey, P. J. 1978, A\&A, 64, 139

Osten, R. A., Ayres, T. R., Brown, A., Linsky, J. L., \& Krishnamurthi, A. 2003, ApJ, 582, 1073

Phillips, K. J. H., Fawcett, B. C., Kent, B. J., et al. 1982, ApJ, 256, 774

Reader, J., Sugar, J., Acquista, N., \& Bahr, R. 1994, Opt. Soc. Am. J. B Opt. Phys., 11, 1930

Schulz, N. S., Canizares, C., Huenemoerder, D., \& Tibbets, K. 2003, ApJ, 595, 365

Seely, J. F., \& Feldman, U. 1986, Phys. Scr, 33, 110

Shirai, T., Sugar, J., Musgrove, A., \& Wiese, W. 2000, J. Phys. Chem. Ref. Data, Monograph, 8

Wargelin, B. J., Beiersdorfer, P., Liedahl, D. A., Kahn, S. M., \& von Goeler, S. 1998, ApJ, 496, 1031

Whiteford, A. D., Badnell, N. R., Ballance, C. P., et al. 2002, J. Phys. B Atomic Mol. Phys., 35, 3729

Zeippen, C. J., Seaton, M. J., \& Morton, D. C. 1977, MNRAS, 181, 527 